\title{
Measurements from an Aerial Vehicle: A New Tool for Planetary Exploration
}

\author{
Henry S. Wright ${ }^{1}$, Joel S. Levine ${ }^{1}$, Mark A. Croom ${ }^{1}$, William C. Edwards ${ }^{1}$, Garry D. Qualls ${ }^{1}$, \\ Joseph F. Gasbarre \\ ${ }^{1}$ National Aeronautics and Space Administration; Langley Research Center; 100 NASA Drive; \\ Hampton, VA 23681
}

\begin{abstract}
Aerial vehicles fill a unique planetary science measurement gap, that of regional-scale, near-surface observation, while providing a fresh perspective for potential discovery. Aerial vehicles used in planetary exploration bridge the scale and resolution measurement gaps between orbiters (global perspective with limited spatial resolution) and landers (local perspective with high spatial resolution) thus complementing and extending orbital and landed measurements. Planetary aerial vehicles can also survey scientifically interesting terrain that is inaccessible or hazardous to landed missions.

The use of aerial assets for performing observations on Mars, Titan, or Venus will enable direct measurements and direct follow-ons to recent discoveries. Aerial vehicles can be used for remote sensing of the interior, surface and atmosphere of Mars, Venus and Titan. Types of aerial vehicles considered are airplane "heavier than air" and airships and balloons "lighter than air.". Interdependencies between the science measurements, science goals and objectives, and platform implementation illustrate how the proper balance of science, engineering, and cost, can be achieved to allow for a successful mission. Classification of measurement types along with how those measurements resolve science questions and how these instruments are accommodated within the mission context are discussed.
\end{abstract}

Keywords: Planetary, Exploration, Venus, Mars, Titan, Airplane, Airship, Balloon, VTOL, Helicopter

\section{INTRODUCTION}

Mars, Titan and Venus provide compelling areas for investigation both for understanding the evolution of the solar system as well as searching for habitable environments (previous and existing), and how those environments potentially could sustain life. Development of autonomous investigations of these planetary bodies has been focused on either, flyby or orbital strategies as well as landers and rovers for surface investigations. One of the key items not currently in the inventory of investigative platforms are aerial vehicles. Scientific investigations conducted on Earth routinely use aerial vehicles as either inhabited or uninhabited science gathering platforms. With the improvements in both planetary autonomous operations (rover and orbiter operations) and the demonstrated performance of terrestrial uninhabited aerial vehicles, the capability has now advanced where autonomous aerial vehicles for planetary exploration are credible concepts.

This paper illustrates the types of measurements and missions which could potentially be undertaken using autonomous aerial vehicles to explore Mars (airplane) and Titan (airship). Included in these areas are descriptions of typical science measurements and platform assessments. Each of these design reference missions has been examined through a rigorous systems analysis process to ensure the mission could be realized and that the payload specific accommodation issues have been considered. Candidate missions to Venus have been explored for both airplanes ${ }^{1}$ and balloons. To date, the only aerial vehicle used in planetary exploration (other than on Earth), has been the Russian Vega-2 balloon in $1985{ }^{2}$

\section{CANDIDATE PLANETARY BODIES}

Primary emphasis of the use of aerial vehicles for planetary science and exploration is focused on those planetary bodies with sufficient atmospheric density to sustain flight. In addition to Earth, Mars, Venus, and Saturn's moon Titan are terrestrial bodies with atmospheres capable of sustaining flight. The gas giants (Jupiter, Saturn, Uranus, and Neptune) could also support flight based science; however, the aspects of flight are sufficiently different, a new system level assessment is warranted and not discussed in this paper. Key planetary constants are provided in Table 1. 
Table 1: Comparison of Elemental Constants for Planetary Bodies Capable of Sustaining Flight ${ }^{3}$

\begin{tabular}{|l|l|l|l|l|}
\hline Parameter & \multicolumn{1}{|c|}{ Earth } & \multicolumn{1}{c|}{ Venus } & \multicolumn{1}{c|}{ Mars } & \multicolumn{1}{c|}{ Titan } \\
\hline Distance from Sun & $149.6 \times 10^{6} \mathrm{~km}$ & $108.2 \times 10^{6} \mathrm{~km}$ & $227.9 \times 10^{6} \mathrm{~km}$ & $1,433 \times 10^{6} \mathrm{~km}$ \\
\hline Diameter & $12,756 \mathrm{~km}$ & $12,100 \mathrm{~km}$ & $6794 \mathrm{~km}$ & $5150 \mathrm{~km}$ \\
\hline Mass $(\mathrm{g})$ & $5.98 \times 10^{27}$ & $4.87 \times 10^{27}$ & $6.42 \times 10^{26}$ & $1.35 \times 10^{26}$ \\
\hline Gravity $\left(\mathrm{cm} / \mathrm{s}^{2}\right)$ & 980 & 887 & 370 & 135 \\
\hline Orbital Period & 365.26 days & 224.7 days & 686.7 days & 15.95 days \\
\hline Rotational Period & $23 \mathrm{~h} 56 \mathrm{~m}$ & 243 days (Retro) & $24 \mathrm{~h} 37 \mathrm{~m}$ & 15.95 days \\
\hline Surface Pressure & $1 \mathrm{bar}$ & $92 \mathrm{bars}$ & $0.008 \mathrm{bar}$ & 1.5 bars \\
\hline Surface Temperature & $15^{\circ} \mathrm{C}$ & $457^{\circ} \mathrm{C}$ & $-143^{\circ} \mathrm{C}$ to $17^{\circ} \mathrm{C}$ & $-180^{\circ} \mathrm{C}$ \\
\hline Atmospheric Composition & $\mathrm{N}_{2}: 78 \% ; \mathrm{O}_{2}: 21 \%$ & $\mathrm{CO}_{2}: 96 \% ; \mathrm{N}_{2}: 4 \%$ & $\begin{array}{l}\mathrm{CO}_{2}: 95 \% ; \\
\mathrm{N}_{2}: 3 \% ; \text { Ar: } 2 \%\end{array}$ & $\begin{array}{l}\mathrm{N}_{2}: \mathrm{CH}_{4}:<3 \% \\
\mathrm{CH}^{2}:\end{array}$ \\
\hline Total Surf. No. Density $\left(\mathrm{cm}^{-3}\right)$ & $10^{19}$ & $10^{17}$ & $10^{19}$ \\
\hline
\end{tabular}

The data in Table 1, illustrates the environmental extremes aerial vehicles must survive and exploit while flying on another planet. Reduced gravity aids in flight, however, the environmental extrema, still provide a unique and intriguing operational environment. Venus' near surface extreme pressures and temperatures require a focus on specialized materials and structural concepts, while the extreme density indicates buoyant flight might be the optimum solution. However, the upper atmosphere of Venus (50 to $80 \mathrm{~km}$ above the surface) has conditions which span Earth to Mars, with lower pressures and more reasonable temperatures than the surface. The low atmospheric pressure of Mars indicates buoyant flight will be challenging. On Mars, with its primarily $\mathrm{CO}_{2}$ atmosphere and lower temperature, the speed of sound is about $67 \%$ of that on Earth, so that Mach effects for an airplane are experienced at much lower flight speeds than on Earth. In addition, on both Venus and Mars, the lack of an oxidizer in the atmosphere indicates use of "air-breathing" power or propulsion is not a credible option. Exploration using aerial vehicles has been successfully demonstrated with buoyant (balloons) technology on Venus. ${ }^{2}$ Many airplane ${ }^{4}$ and buoyant ${ }^{9}$ (balloon and airship) options have been proposed for use on Mars as well as Titan. With the current improvements in communications and autonomous operations technologies coupled with the reduced size and power of many instruments used for planetary exploration, aerial platforms have now become credible planetary exploration platforms.

\section{ROLE OF AERIAL VEHICLES}

Autonomous aerial vehicles enable a new class of science and exploration through their unique near-surface perspective and regional-scale coverage capability. Orbital and other remote sensing measurement techniques provide global coverage but at the cost of resolution. Surface measurements provide high resolution and the ground truth needed to calibrate remotely sensed measurements, but they come at the expense of coverage and regional perspective.

Interactions between the atmosphere and the surface cannot be adequately addressed with either orbital or surface assets as the measurements either include the entire atmospheric column or they are too low in the atmosphere scale height to adequately assess the general processes. Aerial vehicles provide a means to measure processes and phenomena previously unattainable to the planetary scientist. Further, aerial vehicles can increase the overall efficiency of the exploration of the solar system since they cover regional-scale areas at high resolution. The most promising sites for future ground-based missions can be screened and selected with increased confidence as to their value.

\subsection{Aerial Vehicle Types}

For this paper, aerial vehicles are divided into "lighter than air" and "heavier than air" categories; balloons and airships represent the former while powered airplanes represent the latter. Aerial vehicles also considered for use in planetary exploration include vertical takeoff and landing (VTOL), gliders, and ballistic type vehicles. The VTOL's are typically thought of as helicopters. These technologies rely on a propeller or lift fan to provide the lift needed to sustain flight. This class of vehicle can perform measurements while in flight, but the majority of the studies consider this vehicle as a means to combine the science of a lander with the mobility of an aerial vehicle to essentially move the science platform to a new surface location. Gliders have been proposed and considered for many Mars missions as a simple means of performing aerial investigations. Implementation risk is reduced for these types of platforms that lack a propulsion system; however, the flight range is typically limited to 40 to $80 \mathrm{~km}$ with no sustainable altitude control. Since the majority of the needs of the planetary science community require greater range and control in all dimensions, gliders are not further considered in this study. 


\subsection{Measurement Types from Aerial Vehicles}

Aerial vehicles provide an ideal platform for planetary exploration filling a unique measurement niche between orbiters and landers. Aerial vehicles can obtain in situ and remote measurements of terrain and geological features inaccessible to investigation by landers and rovers, such as canyons, valleys, and volcanic and impact craters. Unlike orbiters and landers, aerial vehicles are capable of precision $(\sim 5 \mathrm{~km})$ high resolution surveys covering hundreds of kilometers using only on-board navigation aids. With the addition of off-board navigation aids (from orbit or the surface), this precision can be dramatically improved. Future development of autonomous guidance algorithms will enable the ability to alter the flight path and altitude based on feed back from the science measurements. Certain atmospheric component concentrations and isotopic ratios can only be measured in-situ. By measuring these parameters over a regional-scale, sources and sinks can be sought out. The coupling between the surface and atmosphere and the water cycle on Mars as well as the methane cycle on Titan can be better understood. Improving the resolution of magnetic survey measurements and other potential fields is a capability which can only be provided through aerial vehicles.

The instrument selection for the aerial vehicle is driven by the science measurement needs which produce instrument performance requirements. These performance requirements may be met by current instrument technologies or may require technology development to meet the requirements of mass, volume and power dictated by the aerial vehicle platform. Since the instrument is highly coupled to the aerial platform, the performance of each system is highly coupled. There are many instruments that are currently used in aerial vehicles for Earth Science, planetary probes and satellites that can be adapted for use on planetary aerial vehicles.

\subsubsection{Atmospheric Characterization}

Measurements from aerial platforms are ideal for studying and characterizing the atmosphere of other planets. Extended range, in-situ surveys with high precision measurements of the atmosphere are possible. Aerial vehicles can uniquely perform measurements over specific sites at predetermined altitudes for a desired science target area can be performed. High-resolution measurements of gas composition, isotopes and aerosol characterization are ideally suited to the aerial platform. Both direct sensing and remote sensing instruments may be employed. Direct sensing instruments such as particle detectors, mass spectrometers, and gas chromatographs sense the atmosphere at the location of the aerial platform. Remote sensing instruments such as imagers, radars, radiometers, polarimeters, photometers, atmospheric spectrometers, and altimeters are able to perform measurements at remote distances. Volume, mass and power for many passive instruments can be accommodated with current aerial platform concepts, whereas, active instruments including radars and remote laser based measurements currently require additional development to reduce their mass and power requirements for use on autonomous aerial vehicles. Each of these measurement techniques has already been demonstrated on Earth based aerial platforms thus illustrating the capability of collecting high quality science data.

\subsubsection{Surface and Interior Characterization}

Many studies of planetary bodies require measurements of the surface at higher resolution than can be obtained from orbiters. Planetary geology requires instruments that make measurements of magnetic properties, mineralogy, and imagery. The studies sometime require precise flight tracks at predetermined altitudes. Analyzing the magnetic properties of the surface of Mars is a good example of how an aerial platform can provide high resolution data needed to understand the origins of the magnetism and crustal evolution. This measurement strategy will provide the needed resolution to understand the structure and source of Mars enigmatic remnant crustal magnetism.

High resolution imaging and spectroscopy can be performed from aerial platforms. Terrestrial investigations using aerial platforms have successfully been used as high resolution imaging platforms since almost the inception of modern flight. By virtue of the proximity to the surface, the increased resolution can be achieved with what would be a low or medium resolution image from orbit thus further reducing the developmental complexity.

Many instruments that are currently being used to study the surface of the earth can be employed on aerial vehicles to study planetary bodies. Miniature grating and acoustic-optic spectrometers can make detailed spectral measurements of the surface and are well suited to an aerial platform. Hyperspectral and synthetic aperture radar (SAR) can also be used to characterize the surface but require extensive data handling. Spectrometers, hyperspectral instruments and SAR's produce large amounts of data that can drive the design of data transmission system. Fiber based laser altimeters are well suited for topography measurements and may also be used to provide 3-D images of the surface. 


\subsubsection{Calibration}

Instruments on aerial vehicles must remain calibrated for extended amounts of time (months to years) before reaching their destinations. Methods to enable on-board calibration including stable onboard sources and using external sources such as sunlight must be developed and are specific to the vehicle and instrument design. Since the instruments on the platform are packaged within a sealed aeroshell, designs to minimize contamination and methods to verify instrument operation provide an additional instrument accommodation requirement. Providing calibration targets within the field of view is a common technique employed for imagers. If the there are no locations to place a calibration target, then other techniques must be used. Instruments such as mass spectrometers can use the residual gas within their chambers as a calibration gas source. Accommodating the calibration needs is a critical aspect of accommodating science instruments to ensure return of high quality science data.

\subsection{Exploration of Venus}

Aerial exploration of another planet has been successfully demonstrated at Venus by the Russian space agency IKI in cooperation with the French space agency CNES in $1985 .^{2}$ A small superpressure helium balloon was carried on each of the two Russian Vega Venus probes, launched in 1984. The two balloons with their science payloads were deployed on 11 June 1985, and 15 June 1985. The first balloon failed after only 56 minutes, but the second operated for 47 hours.

The $3.5 \mathrm{~m}$ diameter superpressure balloons were inflated following a direct entry into the atmosphere of Venus. They then floated upward a few kilometers to their equilibrium altitude of $54 \mathrm{~km}$.. At this altitude, pressure and temperature conditions of Venus are similar to those of Earth, though the planet's winds moved at hurricane velocity and the carbondioxide atmosphere is laced with sulfuric acid, along with smaller concentrations of hydrochloric and hydrofluoric acid. With a gross mass of $25 \mathrm{~kg}$ and a $5 \mathrm{~kg}$ instrument payload, Vega 2 survived and transmitted data for almost 47 hours (60 hour design life). Primary science measurements were temperature, pressure, wind speed, light levels, and aerosol density with a sampling rate of once every 75 seconds.

\subsection{Comparison with Orbital and Surface Platforms - Payload Mass}

Aerial platforms are an efficient means of delivering science payloads when compared to other platforms used for planetary science. Figures 1(a) and 1(b) illustrate the science payload mass fraction capability of various interplanetary missions. Extended mission life is a key concern for orbital platforms, consequently, significant attention is paid to the propellant quantity and its use throughout the mission. Rovers have a significant amount of its mass attributed to the drive and suspension systems. Landers have a similar longevity concern as orbiters, as well as the need for safely reaching the surface. Aerial platforms are no different in that they must attribute some of their mass to the schema for remaining aloft as well as maintaining the needed control and orientation. One unique feature of the airplane is that an increase in science payload mass can be accommodated by removing propellant (though not a 1:1 relationship). Two of the aerial platforms have been extensively studied with a large amount of development and risk reduction hardware built, however, they are not approved missions (ARES and Titan). One of the orbital missions (MRO) is an approved mission slated for launch in 2005.

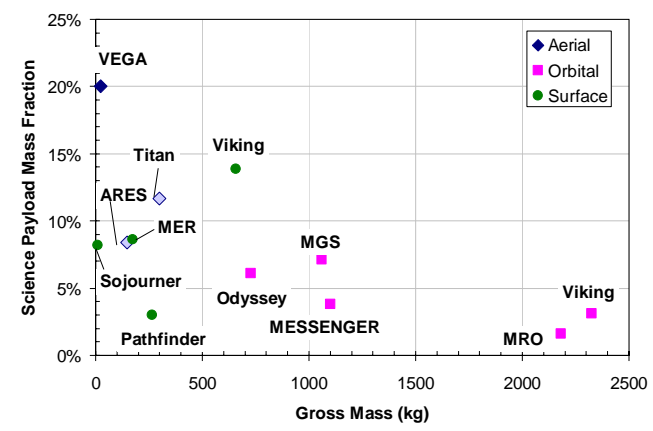

(a) Gross Mass Comparison

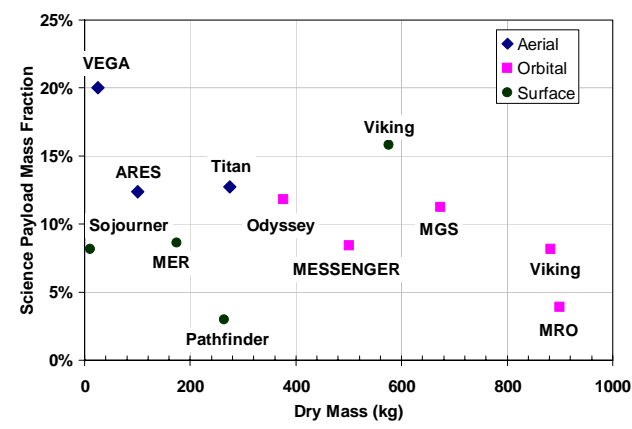

(b) Dry Mass Comparison

Figure 1: Comparison of Science Payload Mass Fraction for Various Platforms 


\section{MARS EXPLORATION}

Exploration of Mars is a significant element of the overall NASA solar system exploration strategy. Recent investigations have definitively determined water stood on the surface of Mars for extended periods of time. Other measurements have hinted at the potential for methane to exist in the atmosphere of Mars. NASA's new exploration strategy brings together the robotic scientific exploration and the human exploration of Mars. These elements have all combined to form a totally new and even more focused need for exploration of Mars. Each platform and measurement type has a role in this overall exploration strategy. Orbiters will continue to be a vital link for exploration. Increased desire to put assets on or near the surface will further enhance our understanding of Mars. Aerial platforms will provide a means of exploring areas where landers and rovers cannot go serve as a long range rapid scout and provide the means to make measurements within the first scale height of the atmosphere. Collectively, this integrated investigative strategy will unveil a totally new and as yet undiscovered Mars.

An airplane mission over Mars will be used as an illustrative example of a potential mission for exploring Mars. This reference mission will highlight the breadth of the potential science goals as well as the types of accommodation and performance requirements achievable with this type of investigation.

\subsection{Science Goals and Measurements of Airplane Reference Mission}

Exploration of Mars using an airplane has been extensively studied. The reference airplane mission used here is the Aerial Regional-scale Environmental Survey of Mars (ARES). ${ }^{4}$ The ARES platform provides simultaneous in-situ and remote measurements of the atmosphere, surface, and interior of Mars from an altitude of 1-2 kilometers above the surface covering regional-scale distances (100s of kilometers). ARES bridges the scale and resolution measurement gaps between the global-scale, lower resolution provided by orbiters and small-scale, higher spatial resolution provided by landers and rovers. Moreover, ARES can survey scientifically interesting terrain that is inaccessible to both landers and rovers. The key ARES scientific themes are: crustal magnetism and near-surface atmospheric chemistry.

\subsubsection{Crustal Magnetism}

The MGS discovery of crustal magnetism in the ancient southern highlands of Mars, a planet without a global dipole magnetic field, was a significant and totally unexpected discovery. ${ }^{5}$ This discovery indicates that Mars had a global magnetic field at some time in its early history produced by an active core dynamo. MGS measurements of the crustal magnetism obtained during the aerobraking phase of the mission at a periapsis altitude of only $100 \mathrm{~km}$ exhibited significantly more spatial detail and information than the MGS measurements obtained at $400 \mathrm{~km}$ above the surface (Figure 2). The spatial resolution or the "imaging" size of crustal magnetic features increases directly as the distance of the magnetometer to the source decreases. Magnetic measurements from satellite altitudes constrain only the product of magnetization and the magnetic layer thickness, for example, at altitudes over $100 \mathrm{~km}$, a $30 \mathrm{~km}$ thick layer of magnetic material with a volume magnetization of $20 \mathrm{~A} / \mathrm{m}$ produces essentially the same signal as a $3 \mathrm{~km}$ thick layer of magnetic material with magnetization of $200 \mathrm{~A} / \mathrm{m}^{6}$

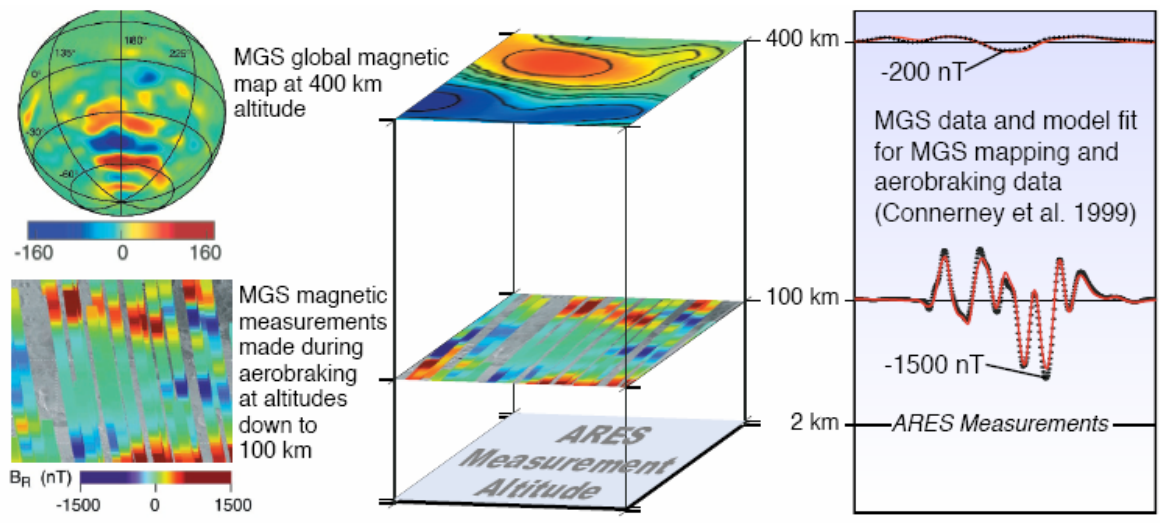

Figure 2: MGS observations at 400 and $100 \mathrm{~km}$ reveal E-W lineations in the ancient terrain of the Southern Highlands. Observations at lower altitudes reveal smaller spatial scale features. An airplane provides measurements at the scale to understand the geologic origin of the magnetic anomalies and the crustal evolution record. 
The only way to "image" the crust magnetically with higher spatial resolution is to measure crustal magnetism closer to the source. The magnetic field produced by variations in magnetization of the crust at small spatial scales is essentially "damped out" at greater altitudes above the crust. Altitude acts as a filter, e.g., the most prominent magnetic variations measured at altitude will be those that correspond to spatial scales comparable to the measurement altitude. High altitude measurements provide information at long spatial scales and low altitude measurements provide information about magnetic variations at small spatial scales.

Study of surface mineralogy, magnetic properties and surface topography all require accurate and predetermined sensor placement (altitude and location). For example, in the figure above, a grid pattern must be used to provide a magnetic survey of the area needed to understand the sources and sinks of the magnetic field. An aerial platform is ideally suited to make this measurement having the ability to fly parallel flight tracks at a fixed altitude.

\subsubsection{Near Surface Atmospheric Chemistry}

The atmosphere of Mars, like the atmosphere of Earth, is a very complex chemical system that regulates the climate, the surface and the general habitability of Mars. Most of our present-day knowledge about this complex chemical system is based on measurements of the major atmospheric gases: carbon dioxide, nitrogen and argon. There are no measurements of the concentration and possible variability of trace atmospheric gases in the near-surface atmosphere over regional-scale distances.

The surface of Mars is a source (e.g., water vapor and gases of possible biogenic and volcanic origin) and a sink (e.g., the surface deposition of chemically active gases) of atmospheric gases. Measurements of atmospheric gases at an altitude of 1-2 km above the surface over regional-scale distances will permit measurements of the chemical coupling between the atmosphere and the surface. Since many chemically-active atmospheric gases are very short-lived, they can only be detected in close proximity to their surface sources such as the diffusion of subsurface water into the atmosphere and a whole variety of gases of possible biogenic and volcanic origin.

One of the important Viking Lander discoveries was that the surface of Mars, unlike the Earth, is very chemically reactive. ${ }^{7}$ It has been hypothesized that the high surface chemical activity of Mars is due to the surface deposition of chemically-active gases, such as hydrogen peroxide $\left(\mathrm{H}_{2} \mathrm{O}_{2}\right)$ and ozone $\left(\mathrm{O}_{3}\right)$. Hydrogen peroxide is a photochemical product of water vapor. By obtaining simultaneous measurements of the concentration of water vapor and its photochemical products, the role of water vapor in the Mars atmospheric chemical cycle can be determined (Figures 3a and 3b). The atmosphere of Mars is a very complex and poorly defined chemical system. The significance of chemically active gases, such as hydrogen peroxide and ozone, over regional-scale distances in the near-surface atmosphere of Mars will be identified and characterized.

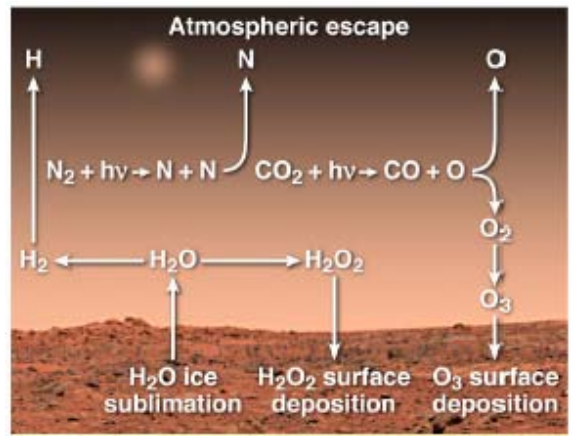

(a) Water Cycle

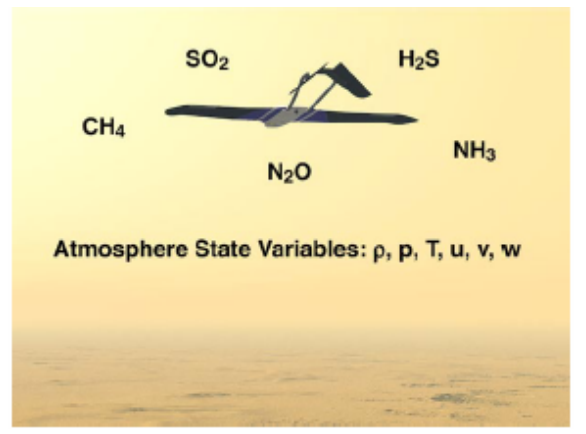

(b) Biogenic and Volcanic Gases

Figure 3: Atmospheric Investigation Areas - Water Cycle of Mars and Search for Gases of Biogenic Origin

\subsection{The Airplane as an Aerial Platform for Planetary Exploration}

Aerial exploration of Mars provides a unique perspective and the ability to collect data in the only achievable manner for specific scientific goals. Use of an airplane is explored here to illustrate both the science collecting capabilities as well as the readily achievable accommodation capabilities. The ability to collect a significant quantity of meaningful science lies in the platform's capacity to carry a suitable science instrument payload to a scientifically interesting target and across a scientifically significant range. A reference set of science goals, objectives, and measurements ${ }^{4}$ has been 
coupled with an airplane to produce an integrated design mission concept which can be performed within the cost and development constraints inherent in the Mars Scout mission class. ${ }^{8,10}$ The ARES mission uses a rocket powered airplane to fly 500 to $680 \mathrm{~km}$ over Mars at an altitude of $1.5 \mathrm{~km}$ above the surface. This mission is illustrated in Figure 4. Many of the challenges facing such a craft stem from the comparatively low density of the Mars atmosphere. After a ballistic entry, the airplane is extracted from the aeroshell, unfolds itself, and performs a transition maneuver from the high speed dive to steady level flight. Recent demonstration of this critical enabling step, through high-altitude flight testing, illustrates the credibility of the platform to be ready for near term implementation. ${ }^{11}$

After performing this transition maneuver, the airplane autonomously flies a preprogrammed flight path which is illustrated in Figure 4. Measurements from the Magnetometer are continuously collected and correlated with the attitude of the airplane. The ARES aeromagnetic survey requires precision flight path control in all axes over a wide expanse, but is particularly challenging on Mars in the horizontal plane. With no global magnetic field and no GPS infrastructure at Mars, accurate ground navigation relies on precise inertial knowledge, which translates into increased mass of airplane support instrumentation. The Mass Spectrometer uses a batch processing mode to collect uncontaminated atmospheric samples across a minimal traverse at the specified altitude. The Mass Spectrometer measurement strategy is such that all of the measurements are performed prior to the first turn of the airplane to preclude contamination of the sample from the rocket effluent. Flight path trades in the presence of winds-aloft modeling and complicated computational aerodynamic analyses are used to satisfy this requirement. Point Spectrometer measurements are continuously collected and processed over the course of the flight. As this is a secondary, or context instrument, its data has lower priority and is transmitted when sufficient bandwidth exists. Context images are collected every 10 seconds to provide a 20\% image overlap, thus providing a continuous photographic record of the flight path. Influence of each of these instruments on the airplane requirements is illustrated in Table 2.

Table 2: Instrument Influences

\begin{tabular}{|l|c|c|c|c|c|c|c|c|}
\hline \multirow{2}{*}{ Instrument } & Vertical & \multicolumn{2}{|c|}{ Lateral } & & \multicolumn{2}{c|}{ Other } \\
\cline { 2 - 9 } & Altitude & $\begin{array}{c}\text { Relative } \\
\text { Navigation }\end{array}$ & $\begin{array}{c}\text { Absolute } \\
\text { Navigation }\end{array}$ & Speed & Stability & LTST & Range & $\begin{array}{c}\text { Mag. } \\
\text { Clean }\end{array}$ \\
\hline Magnetometer & $\mathrm{X}$ & $\mathrm{X}$ & & & & & $\mathrm{X}$ & $\mathrm{X}$ \\
\hline Mass Spectrometer & $\mathrm{X}$ & & $\mathrm{X}$ & & & $\mathrm{X}$ & $\mathrm{X}$ & \\
\hline Point Spectrometer & $\mathrm{X}$ & & & $\mathrm{X}$ & $\mathrm{X}$ & $\mathrm{X}$ & & \\
\hline Context Camera & $\mathrm{X}$ & & & $\mathrm{X}$ & $\mathrm{X}$ & $\mathrm{X}$ & & \\
\hline Video Camera & $\mathrm{X}$ & & & & & & & \\
\hline
\end{tabular}
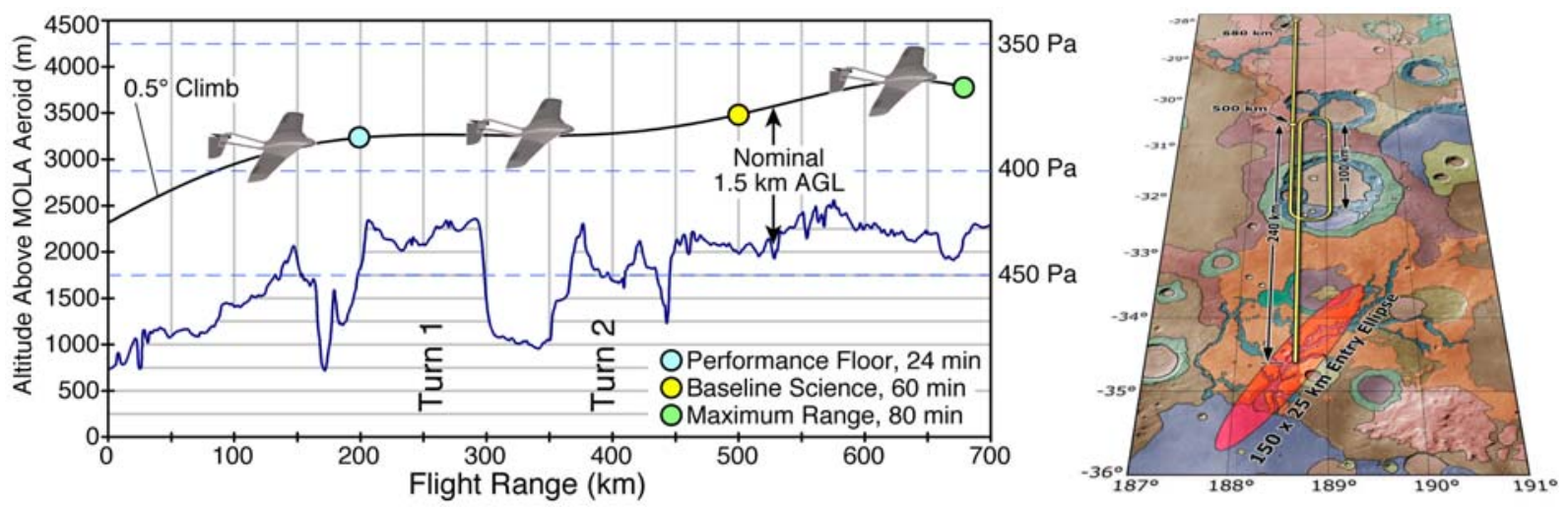

Figure 4: ARES Flight Profile

(Flight Speed: 143 m/s; Wing Span: 6.25 m; Wet Mass: 149 kg; Science Payload: 12.5 kg)

Even though the response to disturbances are minimized by design characteristics, knowledge of the actual attitude and position of the sensor is needed to correlate with the sensor measurement as described next. Imaging-science goals, such as those enabled by the ARES context camera and point spectrometer, impose stability and speed constraints on the vehicle. To serve as an adequate science imaging platform, the sensors must be stabilized or the airplane must be sufficiently stable. The most efficient approach is to ensure airplane stability, even against the poorly understood turbulence and gust environment. Generally, the vehicle should support sub-pixel smear stability. The smear distance at the ground of a nadir-pointing imager is composed of a linear velocity term (proportional to speed and exposure duration) and a rotational term (proportional to angular rate, exposure duration, and height). A fully non-linear 6-DOF 
simulation accounting for all the translational and rotational aerodynamic and motion terms along with a complete description of the flight control system capabilities is needed to accurately assess the performance (Figure $5 \mathrm{~b}$ ). ${ }^{12}$

\subsection{Instrument Accommodation}

Accommodation of the science instruments including providing the needed airplane attitude or state correlation information is readily achievable. In addition to the accommodation needs defined in Table 2, thermal, vibration, stability limitations, and attitude determination accommodation aspects are also considered.

Simple strap down mounts for all of the instruments with modest vibration and thermal isolation is sufficient for this class of mission. Thermal requirements are primarily driven by the spectrometer portion of the Point Spectrometer. Excessive thermal excursions could cause thermal expansion or contraction of the spectrometer thus corrupting the ray trace. Thermal expansion is controlled with insulating mounts and a thermal housing around the telescope optics. Thermal control of the other instruments and subsystems (computers, radios, etc.) is provided through traditional methods such as insulation and strap-on heaters. Thermal analysis indicates overheating is not an issue.

Vibrations stemming from the airplane and its subsystems, atmospheric conditions, the launch environment (vibration, shock, accelerations) are all addressed as part of the operational performance analysis. The primary vibration source for instrument accommodation is the propulsion system. Vibration could result in smeared images (Context Camera and Point Spectrometer) as well as increased uncertainty in attitude based measurement sets (e. g., the magnetometers). Both the Context Camera and the Point Spectrometer use Charge Coupled Device (CCD) as the primary photon collection element. With CCD integration times of 7 to 10 milli-seconds, and a maximum allowable smear limit of $1 / 2$ of a pixel, then it is possible to assess the ability to provide a platform with sufficient stability. The design reference mission uses a bi-propellant rocket operating in a periodic operational mode (3 seconds on and 3 seconds off) for its propulsion system. The peak acceleration change is approximately $0.4 \mathrm{~m} / \mathrm{s}^{2}$ (or $4 \%$ of one Earth-g). This diminutive axial acceleration is below the measurement threshold of the majority of this class of science measurements (Figure 5a). The instruments themselves and the non-imaging data sets, are not overly sensitive to vibration.

Platform stability focuses on how well the airplane responds to the local environment to maintain the desired attitude and state. Assessing the winds and turbulence levels on Mars is performed using statistical (Monte-Carlo) techniques. Mars, like Earth, has variable a variable environment such that models are used to provide the bounds, or extrema, of conditions to be considered. Platform stability influences primarily the imaging instruments as the sampling rate of both the Mass Spectrometer and the Magnetometer is significantly shorter than the anticipated airplane kinematic response to an input. Figure 5(b) highlights the platform stability for the airplane as a function of pixel smear when it is subjected to turbulence levels which are equivalent to Severe Turbulence on Earth. Figure 5(a) illustrates the baseline pixel smear due to the steady cruise speed ( $70 \%$ of the total pixel smear). ${ }^{12}$

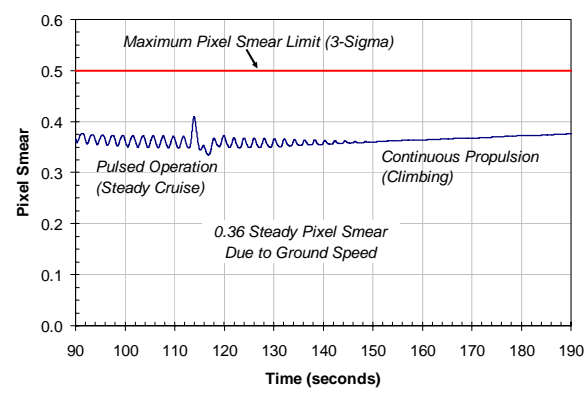

(a) Ground Speed + Propulsion

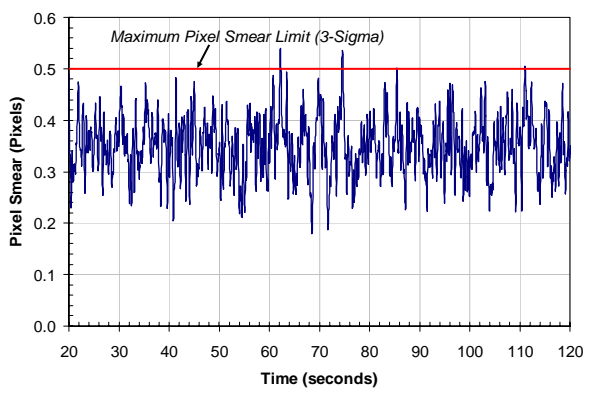

(b) Ground Speed + Propulsion + Turbulence

\section{Figure 5: Pixel Smear Induced by Flight and Environmental Influences - From 6 DoF Simulation}

One of the key instrument accommodation elements is the platform state determination of both the attitude relative to the planet as well as the deformed state of the airplane. Since the primary structural design driver is the launch and entry loads, the design reference platform is inherently stiff and does not experience any significant deformations or deflections during the flight. Vehicle state in terms of its altitude, heading, and apparent ground speed are provided through the airplane's navigational sensor suite. A radar altimeter and the static pressure provide the correlated altitude. The air data system provides the air relative flight speed, while an inertial measurement unit (IMU) provides the planet 
relative flight speed and rotation rates. Coupling this data provides the necessary data set to determine the planet relative state so that each of the instrument data sets can be correlated.

\subsection{Mars Airplane Summary}

A design reference mission, including science instruments, and their accommodating aerial platform has been developed. Assessments of the mission's ability to collect and correlate scientifically meaningful measurements show high data quality is achievable on a moving platform with an active thrust control that will significantly improve our understanding of Mars in ways previously unattainable.

\section{TITAN EXPLORATION}

In the search for life outside the Earth, Titan, the largest moon of Saturn, holds a very unique position. Titan is larger in diameter than Mercury, larger and more massive than Pluto, and slightly smaller than Mars. Titan has emerged as a puzzling and very complex world with a strange atmosphere and surface. Like the terrestrial planets, Titan has a solid surface and a density that suggests the moon is composed of a mixture of rock and ice in almost equal amounts. Titan may provide the details to explain how life formed on Earth very early in its history.

\subsection{Science Goals and Measurements}

Key observations of Titan include a Voyager flyby and Hubble Space Telescope observations. Commencing in July 2004, the Cassini-Huygens mission began the latest investigation of Titan and Saturn with the Huygens probe planned to be delivered to Titan in January 2005.

\subsubsection{The Atmosphere of Titan}

Titan's atmosphere may hold answers to the evolution of prebiological chemistry on the early Earth. ${ }^{13,14,15}$ Titan is surrounded by a thick, opaque orange-colored atmosphere with a surface pressure of 1.5 bars. Visible imaging of the surface of Titan from orbit is not feasible due to the thick layers of opaque haze and clouds in the atmosphere. ${ }^{16}$ Upcoming data from the Huygens probe should aid in characterizing the opacity as a function of altitude.

Molecular nitrogen $\left(\mathrm{N}_{2}\right)$ is the overwhelming constituent of the atmosphere (about $95 \%$ by volume), with smaller amounts of methane $\left(\mathrm{CH}_{4}\right)$ and molecular hydrogen $\left(\mathrm{H}_{2}\right) .{ }^{17}$ The stability of methane in Titan's atmosphere is puzzling, since the atmospheric lifetime of methane is controlled by its destruction by solar ultraviolet radiation, which is short on cosmic timescales (about $10^{7}$ years). The cloud and haze are sufficiently thick that ultraviolet radiation cannot penetrate to the troposphere, hence, atmospheric methane on Titan appears to be resupplied by a possible surface reservoir.

Photochemical and chemical reactions initiated by methane (and nitrogen) leads to the production of numerous hydrocarbons of increasing chemical complexity, beginning with ethane, hydrogen cyanide, etc., and leading to complex organic compounds such as purines, pyrimidines, and aldehydes, believed to be the chemical precursors of the first living systems on Earth. ${ }^{13,14,15}$ The dominance of nitrogen on Titan, gives rise to the rich coupled chemistry between nitrogen and carbon. The variety of nitrile species on Titan appears to be unique in the Solar System. It is generally believed the atmosphere of Titan is similar to the Earth's primordial atmosphere, the atmosphere that produced the complex organic molecules that led to the formation of living systems on Earth in its early history.

The early history and evolution of the atmosphere of Titan is a key scientific question. Due to its low gravitational attraction, Titan can easily lose atomic $(\mathrm{H})$ and molecular $\left(\mathrm{H}_{2}\right)$ hydrogen to space. With the loss of atomic and molecular hydrogen, the production of complex hydrocarbons becomes irreversible. For example, 96\% of the dissociation of methane results in the production of complex hydrocarbons on Titan. The corresponding efficiency for Jupiter is only $66 \% .{ }^{17}$ Measurements of the isotopic ratios of the carbon, hydrogen, nitrogen and chemically inert gasses will provide important information on the evolution of the atmosphere of Titan.

\subsubsection{Meteorology and Circulation}

Titan's "hydrological" cycle involving the condensation, precipitation and evaporation of hydrocarbons may resemble the water hydrological cycle on Earth. The Hubble Space Telescope (HST) mapped light and dark features over the surface during a complete 16-day rotation period. The HST tracked a bright area surface feature some 2500 miles across. Calculations indicate that Titan has roughly 100 times more latent heat available for fueling weather than does 
the Earth's atmosphere. Recent observations of the presence of clouds that form at the tropopause are evidence for hurricane-sized cloud systems. The nature and formation of the clouds, the origin of the large storm systems, and the effects of latent heat on cloud formation and atmospheric circulation are unknown. ${ }^{16,18}$

\subsubsection{The Surface}

Ethane/methane lakes or oceans would be similar to the primordial sea on Earth that led to the production via polymerization reactions, of the first living systems on the early Earth. It has also been hypothesized that the tropopause acts as a "cold trap," where gaseous organic compounds condense out of the atmosphere and are, removed from the atmosphere and then deposited onto the surface. For example, ethane precipitates out of the atmosphere onto the surface producing ponds, lakes or oceans of ethane (or ethane/methane). An ethane/methane ocean at the surface may be the source of the re-cycling of methane back into the atmosphere.

\subsection{Design Reference Mission}

Characterizing capabilities and limitations of potential measurement sets has been examined through the use of a Design Reference Mission (DRM). The DRM uses a dedicated orbiter as a science observation and communications relay platform and an airship for in-situ investigations. The basic mission architecture of the DRM is derived from previous mission concept studies. ${ }^{19,20}$ The mission uses a chemical propulsion with Venus and Earth gravity assists followed by aerocapture for the Titan orbit insertion. A near polar $1700 \mathrm{~km}$ altitude orbit is used.

\subsubsection{Airship Description}

One of the most alluring aspects of flying anything on Titan is that it is possible to design an aerial platform which is very similar to what flies on Earth everyday, thereby reducing inherent design risk. It is reasonable to assume that any of three types of powered aerial platforms could be flown on Titan: a helicopter, a fixed-wing airplane, or an airship. Selection of the aerial vehicle for the Titan DRM considered these three platform types. The mission requirements included flying the reference science payload, a maximum vehicle mass of $400 \mathrm{~kg}$, and the goal of surviving for at least 90 days (at a flight speed of $3 \mathrm{~m} / \mathrm{s}$, a circumnavigation of Titan could be accomplished in about 75 days). According to the latest engineering model of Titan's atmosphere, ${ }^{21}$ the variation of density with altitude in the region near the surface $(<50 \mathrm{~km})$ is much smaller than that of Earth's while the density at these altitudes is between 4 and 7 times greater. The dominant winds on Titan are zonal, with a prograde circulation generally observed.

An airplane would use a propeller driven by an electric motor. Due to the higher atmospheric density than Earth, the required propulsion power at even moderate speeds $(>20 \mathrm{~m} / \mathrm{s})$ is excessive. The need for reduced propulsion power resulted in an airplane with long, slender wings (high aspect ratio). This configuration means multiple folds are needed in each wing for aeroshell packaging, thus increasing the deployment risk. Although VTOL vehicles are not specifically considered in this paper, notionally some of these characteristics can be mitigated when considering a helicopter or other VTOL craft. However, VTOL induce even greater design complexity issues and thereby could induce an unwanted amount of risk. In the case of an airship, the density, pressure, and temperature difference influence the velocity of the airship as it relates to the total vehicle drag and the gasbag volume. The resulting airship design has an overall length of $15 \mathrm{~m}$ and a maximum diameter of $3.3 \mathrm{~m}$. Ballonets are used to provide a simple means of altitude control. While the small variation of density with altitude may make holding exact altitude difficult, it will also allow easy access to the surface with minimal variation in gasbag volume. Compared to the airplane, the airship will only be able to move at a fraction of the forward velocity. When considering the airship's ability to station-keep, its relatively simple and straight-forward design, and its ease of surface access (either by landing or a combination of hovering and dropping a retractable probe), it is clear the airship is the optimum choice for Titan aerial investigations. A summary of the advantages and disadvantages of each of the three platforms can be seen in Table 3.

Table 3: Aerial Platform Comparison

\begin{tabular}{|l|l|l|}
\hline & \multicolumn{1}{|c|}{ Advantages } & \multicolumn{1}{|c|}{ Disadvantages } \\
\hline Airplane & Rapid surface coverage, maneuvering in winds & $\begin{array}{l}\text { Limited landing capability, no station-keeping } \\
\text { ability, packaging issues, large propulsion power }\end{array}$ \\
\hline Helicopter & $\begin{array}{l}\text { Station-keeping ability, multiple landings } \\
\text { possible, adequate surface coverage }\end{array}$ & Complex, high power system, packaging issues \\
\hline Airship & $\begin{array}{l}\text { Station-keeping, multiple landings or surface } \\
\text { interactions possible, comparatively low power, } \\
\text { adequate surface coverage }\end{array}$ & $\begin{array}{l}\text { Navigation can be subject to winds, landings not } \\
\text { trivial, packaging issues }\end{array}$ \\
\hline
\end{tabular}


The airship is delivered to Titan inside of an entry aeroshell. As the aeroshell descends on a parachute, the airship is extracted and inflated using helium. Near the desired float altitude $(5 \mathrm{~km})$, the airship is released and starts the mission. Most of the helium tanks are jettisoned with the backshell to reduce the airship float mass. The propulsion system uses two gimbaled propellers to provide forward flight, yaw control, and to assist in station-keeping. Stirling Radioisotope thermoelectric Generators are used for the propulsion, avionics, and science payload energy source. The gondola of the airship will house the science instrument suite, the internal operating components (avionics, batteries, power distribution system, etc.), and additional reserve tanks for re-inflation of the gasbag. It is assumed that occasionally helium will be vented from the gasbag to allow close encounters with the surface or possible landings. A UHF relay link to the orbiter is the primary communications path. Navigation of the airship will be by waypoint and IMU inertial reference, possibly updated by the orbiter and/or through the use of a laser altimeter. Overall, this preliminary design should provide a platform for a long duration general survey of most of Titan's surface and atmosphere.

\subsubsection{Science Payload}

Science payloads for the orbiter and the airship have been defined. Science instruments for an orbiter have been selected that will provide global observations of Titan complementing the Cassini-Huygens measurements. The airship platform will be able to provide high resolution measurements of the atmosphere and surface. Preliminary power and mass estimates have been made and are based on previously flown space instruments performing similar measurements (Table 4 for the Orbiter, and Table 5 for the Airship).

Table 4: Orbiter Science Payload

\begin{tabular}{|l|l|c|c|l|}
\hline Instrument & Use & $\begin{array}{c}\text { Mass } \\
(\mathbf{k g})\end{array}$ & $\begin{array}{c}\text { Power } \\
(\mathbf{W})\end{array}$ & Basis \\
\hline Solar Occultation & Atmospheric composition \& isotopic ratios & 2 & 5 & TBD \\
\hline Laser Altimeter & Nature of the surface & 5 & 20 & MESSENGER-MLA \\
\hline Magnetometer & Planetary dipole \& surface magnetism & 1.5 & 2 & MGS \\
\hline UV Spectrometer & $\begin{array}{l}\text { Upper atmos. escape of atomic \& } \\
\text { molecular Hydrogen }\end{array}$ & 10 & 6.5 & Cassini \\
\hline $\begin{array}{l}\text { Visional \& IR } \\
\text { Mapping Spectrometer }\end{array}$ & Cloud \& haze layer, surface & 37 & 21 & Cassini \\
\hline
\end{tabular}

Table 5: Airship Science Payload

\begin{tabular}{|l|l|c|c|l|}
\hline Instrument & Use & $\begin{array}{c}\text { Mass } \\
(\mathbf{k g})\end{array}$ & $\begin{array}{c}\text { Power } \\
(\mathbf{W})\end{array}$ & Basis \\
\hline Imaging System & Surface features, clouds, and haze & 0.4 & 4.5 & Clementine UV/Vis \\
\hline Mass Spectrometer & Atmos. composition \& isotopic ratios & 5 & 7 & Pioneer Venus \\
\hline Haze \& Particle Detector & Cloud particle size \& concentration & 2.5 & 20 & Pioneer Venus \\
\hline Spectrometer & Nature \& composition of the surface & 2.5 & 3 & MESSENGER - MASCS \\
\hline $\begin{array}{l}\text { Sun Seeking } \\
\text { Spectrometer }\end{array}$ & Atmospheric Opacity & 10 & 10 & New \\
\hline
\end{tabular}

\subsubsection{Titan Airship Summary}

An integrated systems study was performed to select the optimum platform for long duration integrated exploration of Titan. The airship and the orbiter with their complementary science payloads provide an unprecedented opportunity for understanding the evolution of Titan and potentially, Earth.

\section{CONCLUSIONS}

Our perspective of Mars, Titan, and Venus will undergo a dramatic shift after the flight over another planetary body of an autonomous aerial vehicle for scientific exploration. Aerial vehicles provide a unique perspective in concert with the ability for regional-scale measurements. Furthermore aerial vehicles provide the optimum method for performing surveys of magnetic, gravimetric, or general resource surveys. Aerial vehicles also provide the only means for investigating the regional-scale interactions between the atmosphere and the surface. Recent and current activities aimed at maturing the use of autonomous airplanes for Mars will enable near term application of this platform. Systems studies indicate the airship is the simplest "first-flight" application for Titan. Succeeding missions will use enhanced versions of the airship or balloon platform as well as the VTOL type platforms to increase the potential for significant interaction 
with the ground. The scientific exploration of the planets will begin a new and exciting era with the use of autonomous aerial vehicles for planetary exploration.

\section{REFERENCES}

1. Colozza, A. Feasibility of a Long Duration Solar Powered Aircraft on Venus. AIAA 2004-5558. ${ }^{\text {nd }}$ International Energy Conversion Engineering Conference; August 16-19, 2004. Providence, RI.

2. Website: http://nssdc.gsfc.nasa.gov/database/MasterCatalog?sc=1984-125A

3. Levine, J. S. Planetary Atmospheres. Encyclopedia of Physical Science and Technology, Third Edition, Volume 12, Academic Press, Inc., San Diego, CA, 2002, 245-273.

4. Levine, J. S.; et. al.: Science From a Mars Airplane: The Aerial Regional-scale Environmental Survey (ARES) of Mars. AIAA 2003-6576. 2003

5. Acuna, M. H.; Connerney, J. E. P.; Ness, N. F.; Lin, R. P.; Mitchell, D.; Carlson, C. W.; McFadden, J.; Anderson, K. E.; Reme, H.; Mazelle, C.; Vignes, D.; Wasilewski, P.; Cloutier, P.: Global Distribution of Crustal Magnetism Discovered by the Mars Global Surveyor MAG/ER Experiment. Science. 284; 790-793.

6. Connerney, J. E. P.; Acuna, M. H.; Wasilewski, P.; Ness, N. F.; Reme, H.; Mazelle, C.; Vignes, D.; Lin, R. P.; Mitchell, D.; Cloutier, P.: Magnetic Lineations in the Ancient Crust of Mars. Science. 284, 794-798.

7. Banin, A.; Clark, B. C.; Weanke, H.: 1992. Surface Chemistry and Mineralogy. Mars . The University of Arizona Press, Tucson, AZ, 594-626.

8. Wright, H. S.; Croom, M. A.; Braun, R. D.; Qualls, G. D.; and Levine, J. S.: ARES Mission Overview: Capabilities and Requirements of the Robotic Aerial Platform. AIAA 2003-6577. 2003

9. Greeley, R.; et. al.: The Mars Aerial Platform (MAP) Concept. AIAA 1996-0335. 1996.

10. Braun, R.D., Wright, H.S., Croom, M.A., Levine, J.S., and Spencer, D.A., "The Mars Airplane: A Credible Science Platform,” IEEE 1260, IEEE Aerospace Conference, Big Sky, MT, Mar. 6-13, 2004.

11. Lede, J. C.; Parks, R.; Croom, M. A.: High Altitude Drop Testing in Mars Relevant Conditions for the ARES Mars Scout Mission. AIAA 2003-6609. 2003

12. Kenney, P. Sean; Croom, Mark, A.; Simulating the ARES Aircraft in the Mars Environment, AIAA 20036579. $2^{\text {nd }}$ AIAA Unmanned, Unlimited Conference, San Diego, CA, Sept. 2993,

13. Levine, J. S.: The Photochemistry of the Paleoatmosphere. Journal of Molecular Evolution, 18, 1982a, 161172.

14. Levine, J. S. ;Augustsson, T. R.; and Natarajan, M.: The Prebiological Paleoatmosphere: Stability and Composition. Origins of Life, 12, 1982b, 245-259.

15. Levine, J. S.: The Photochemistry of the Early Atmsophere. The Photochemistry of Atmospheres: Earth, The Other Planets, and Comets (J. S. Levine, Editor), Academic Press, Inc., San Diego, CA, 1985, pp. 3-38.

16. McKay, C. P.; Lorenz, R. L.; Lunine, J. I.; 1999. Analytic Solutions for the Antigreenhouse Effect: Titan and the early Earth. Icarus, 137, 56-61.

17. Yung, Y. L. and Demore, W. B., 1999: Photochemistry of Planetary Atmospheres. Oxford University Press, New York.

18. McKay, C. P., et. al., 2001 Physical Properties of Organic Aerosols and Clouds on Titan. Planetary and Space Science, 49, 79-99.

19. Lockwood, M.K., “Titan Aerocapture Systems Analysis,” AIAA-2003-4799, 39 ${ }^{\text {th }}$ AIAA/ASME/SAE/ASEE Joint Propulsion Conference \& Exhibit, Huntsville, Al, July 20-23, 2003.

20. Bailey, R., Hall, J., Spilker, T., “Titan Aerocapture Mission and Spacecraft Design Overview,” AIAA-20034800, 39 $9^{\text {th }}$ AIAA/ASME/SAE/ASEE Joint Propulsion Conference \& Exhibit, Huntsville, Al, July 20-23, 2003.

21. Yelle et al.: "Engineering Models for Titan’s Atmosphere”, in Huygens Science, Payload and Mission, ESA SP-1177, 1997 